\title{
New HIV vaccine center has Haynes at its helm
}

\begin{abstract}
he he new Center for HIV/AIDS Vaccine Immunology (CHAVI), set up by the NIH's National Institute of Allergy and Infectious Diseases (NIAID), is a consortium of researchers at various universities who will collaborate with the goal of creating an effective HIV vaccine that can be used worldwide. Barton Haynes, a Duke University immunologist and director of the Duke Human Vaccine Institute, is CHAVI's new director.

Haynes will work with foremost investigators Norman Letvin, Joseph Sodroski, George Shaw, and Andrew McMichael. Together they competed with and defeated 3 other potential teams to form the scientific leadership group for CHAVI, responsible for the overall work the consortium conducts. The JCI spoke to Haynes about his new position, the future of AIDS vaccines, and how this
\end{abstract}

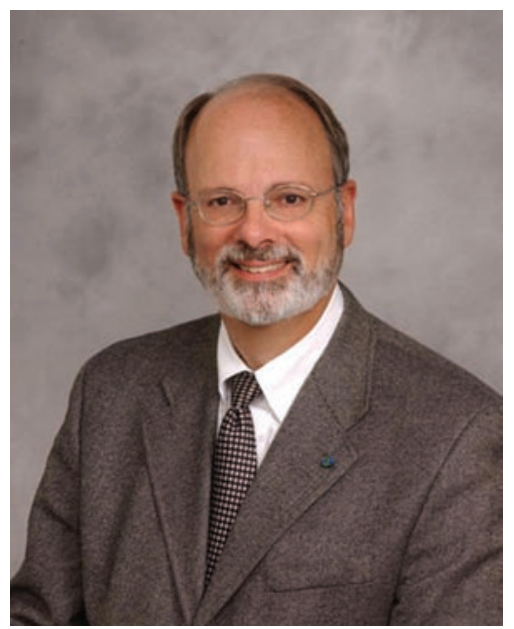

Barton Haynes, new CHAVI director. Photo courtesy of Duke University Medical Center. multimillion dollar endeavor might change the course of the AIDS epidemic.

JCI: How was your team selected for this position?

Haynes: We were selected through a peer review process of an NIH-funded grant.

JCI: How will CHAVI be different from other groups that carry out AIDS vaccine research?

Haynes: CHAVI will be more highly coordinated and will synergize with other groups in open and transparent ways. We will be an integral part of the overall effort of the Division of AIDS at NIAID and the Global HIV AIDS Vaccine Enterprise.

JCI: What do you think are the biggest challenges that AIDS researchers face today?

Haynes: Understanding correlates of protective immunity, overcoming HIV diversity, and [achieving] induction of broadly neutralizing antibodies.

JCI: What do you anticipate will be your biggest challenge in this new endeavor?

Haynes: The first challenge will be to establish the infrastructure for a functional, virtual organization. This will need to include a communications system of phone, video conferencing, and face-to-face meetings to develop and orchestrate functional research teams. Second, the initial focus of research for the CHAVI teams will be an extensive analysis of both the host and the virus during the very earliest stages of acute HIV infection. We will work to enroll a large cohort of acute HIV-infected patients for these studies. Third will be the analysis of those subjects repeatedly exposed to HIV who still appear to be uninfected. We will need to determine if they are truly uninfected, and if so, define the immunologic or genetic factors that contribute to protection. Next will be the challenge to define correlates of protection in nonhuman primates to a virus challenge that is relevant to transmission of HIV in human infection. And finally will be the challenge to translate our discovery efforts to the development of novel vaccine candidates for iterative testing in human trials.

JCI: How do you foresee your typical day?

Haynes: Lots of conference calls, visits to collaborators and advisors, lots of data analysis. Initially, we will spend time setting up administrative infrastructure, and then after the first 4-6 months, will spend more time on technology development and scientific project administration. We have already assembled a crack administrative team that knows how to run such a large and diverse organization.

JCI: What are your 3 top initial goals for CHAVI?

Haynes: Establishing a functional CHAVI organization infrastructure, elucidating the correlates of protective immunity in the context of acute HIV infection and primate infection models, and bringing HIV vaccine candidates to phase I clinical trials in man that, in preclinical studies, induce immune responses at systemic and mucosal sites, and that we believe will be predictive of clinically relevant protective anti-HIV immune responses in man.

JCI: What in your life are you most proud of?

Haynes: My family. And coaching my children's sports teams.

\section{Stacie Bloom}

\section{Parasite genome similarities offer hope for new drugs and vaccines}

I t took nearly 250 scientists from 47 institutions on 6 continents over 6 years to resolve the genetic makeup of 3 deadly parasites that are responsible for causing hundreds of thousands of cases of disease each year. The information, published in 3 papers in the July 15 issue of Science, provides new opportunities for developing new therapies (1-3).

"The most immediate benefit will be a change in the way research in these fields is conducted," said Matthew Berriman, lead author on one of the papers. "With a genome sequence, there's a framework for generating new ideas and rapidly testing them. The ultimate aim of these projects is to accelerate development of vaccines, new drugs, and diagnostics."

African sleeping sickness, leishmaniasis, and Chagas disease are insectborne diseases caused by the parasites 